\title{
Study Personnel Postal Address
}

National Cancer Institute

\section{Source}

National Cancer Institute. Study Personnel Postal Address. NCI Thesaurus. Code C94101.

A contact point used to send physical forms of communication to the study personnel. 